\title{
Parametric synthesis of assembly device for fitting rubber sealing ring into the inner groove of the basic item
}

\author{
Aleksey Tarakhovskiy, ${ }^{1,}$, Sergey Sazonov ${ }^{1}$, Denis Tokarev $^{1}$ and Yuriy Moroz ${ }^{1}$ \\ ${ }^{1}$ Sevastopol State University, 33, Universitetskaya St., Sevastopol, 299053, Russia
}

\begin{abstract}
On the basis of the block diagram of the assembly device technological system a group of morphological matrices of various hierarchy levels may be built. The analysis of the engineering system " the assembly device for assembly Seal of connections " is manufactured. The structure of the engineering system and e ë of units at different hierarchical levels is defined, the links between subsystems are detected. On the basis of the skeleton diagram of the assembly device the group of morphological matrixes, different levels of hierarchy is constructed. The synthesis of variants of structure of the assembly device is manufactured.
\end{abstract}

\section{Introduction}

Analysis of assembly device for assembling the sealing compound (elastic annular item - groove of the hole) shows that the assembly device possesses all the features of the engineering dynamic systems. Considering this problem of synthesis of optimal structures of assembly device can be regarded as a common task of the theory of synthesis of engineering systems, but it also has a number of features related to:

- the requirements to the assembly device;

- a physical feature of the assembly.

\section{Synthesis of assembly device}

Synthesis of assembly device may be configured basing on the use of structural and parametric optimization synthesis methods of engineering systems.

The synthesis problem solving sequence of the assembly device:

1. Formation of a plurality of the assembly device structures.

2. Assessment of structure options by extensional indexes.

3. Evaluation of options in accordance with stochastic processes.

4. Identification of "weak" places in the technological system.

5. Development and improvement of the "weak" places of the system.

6. Evaluation of the system effectiveness considering its verifications.

7. The implementation of the system.

In the first stage of solving the synthesis aim of the assembly device system the structure of the engineering system and its constituent subsystems should be determined. To do this, you must submit morphology (structure) of an engineering system, and then the connections between its elements (subsystems) and their parameters. After carrying out the study at one level (the level of the initial engineering system) analysis then in most cases is required to continue to lower hierarchical levels (at the levels of specific subsystems).

For this purpose, when forming a plurality of structures of different effects on the output indicators as the process system, and the assembly process are taken into consideration as attributes and characteristics of their technological elements. In this case it is advisable to apply a morphological analysis unit, representing the engineering system elements (or its functions) as morphological features.

However, this method is in its pure form can not be used, since the choice of efficient variations does not take into account the actual conditions of the assembly process of sealing compounds. Therefore, morphological analysis should be used as a unit on the first two stages of the synthesis to form a plurality of structures and assessment of options for extensional indexes.

On the basis of the block diagram of the assembly device technological system a group of morphological matrices of various hierarchy levels may be built. To do this in the technological system of the assembly device, some structural or functional characteristic for its morphological features should be highlighted. Each feature may characterize a structural unit of the system or its function or mode of operation of the system, i. e. parameters or characteristics of the system, from which the solution of the problem and the achievement of the main goal depend.

The list of various specific characteristics the expression of technical alternatives for each selected morphological feature is made. Features and their alternatives are presented in tabular form.

\footnotetext{
Corresponding author: atarahovsky@gmail.com
} 
Table 1. Morphological matrix of assembly device for assembling a sealing compound (elastic annular item - groove of the hole)

\begin{tabular}{ll}
\hline Subsystem - assembly mechanism (AM) & \\
\hline subsystem feature & Feature characteristics \\
\hline Deforming body - X1 & assembly device X11 \\
& $\ldots$ \\
& Basic item X1m \\
\hline$\ldots$ & \multicolumn{1}{c}{$\begin{array}{l}\text { n.1 Due to the elastic properties of the elastic } \\
\text { ring item - X51 }\end{array}$} \\
\hline n. Restoring the deformed shape - Xn & $\ldots$ \\
& n.m Due to assembly device - Xnm \\
\hline
\end{tabular}

Similarly matrices for the other subsystems are constructed: the basic item (BI), a elastic ring item (ERI), the basic item of the supplying device (SBI) and feeder of elastic ring item (FERI).

The next step after filling the morphological matrices is a synthesis of options for the structure of the assembly device. To do this, from a variety of technological features of the subsystem - "assembly device" the most applicable ones for the given conditions should be selected.

Suppose we have $M$ types of $n$-th feature of the subsystem, with $m$ - the serial number of the given subsystem, then the variety of the subsystem conditions of "assembly device" can be expressed in the form of a matrix.

$$
\|C \sigma\|=\left\|\begin{array}{cccc}
X_{11} & X_{21} & \ldots & X_{N 1} \\
X_{12} & X_{22} & \ldots & X_{N 2} \\
\ldots & \ldots & \ldots & \ldots \\
X_{1 M_{1}} & X_{2 M_{2}} & \ldots & X_{N M_{N}}
\end{array}\right\|
$$

By analogy, a variety of the subsystems conditions is expressed in the same way in the form of matrices: the basic item (BI), a elastic ring item (ERI), the basic item of the supplying device (SBI) and feeder of elastic ring item (FERI).

Subsystem features from morphological tables are chosen to build graphs for set of possible variants of "assembly device" which is equal to the number of paths in the graph. Figure 1 shows a graph of possible structural variants of the technological subsystem of "assembly device". Graphs of possible variations of other technological subsystems are constructed similarly.

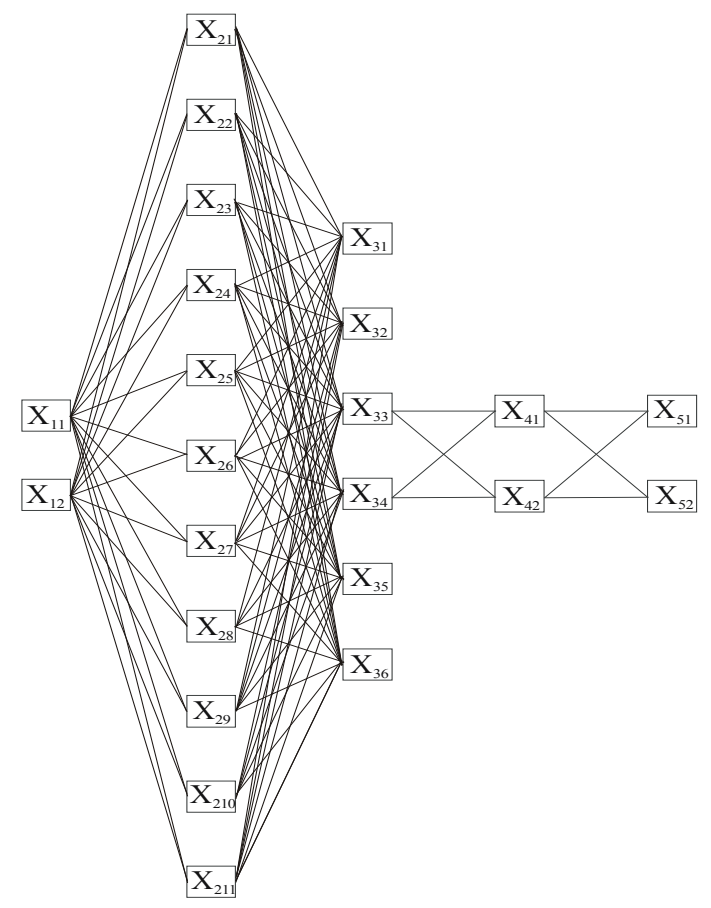

Fig. 1. Graph of possible structural variants of the technological subsystem of "assembly device". 
When considering a large number of variants the solution synthesis should be carried out, starting from the basic elements of choices, including more and more significant features.

Let's carry out a set of technological subsystem variations of "elastic ring items." Made investigations have shown that the most frequent application is being made with sealing compounds with elastic ring items in the form of annular rubber rings of circular cross-section. They differ in design simplicity, a wide versatility, and durability, ease to manufacture, reliability in operation, and are used at wide temperature and pressure ranges. The most appropriate structure of subsystem "elastic ring item " is shown in Figure 2.

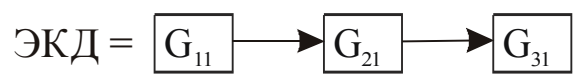

Fig. 2. structure of subsystem "elastic ring item "

where the G11 - kind of ERI (ring); G11 - diametrical sectional view of the ERI (simple round); G11 - number of installed elements (one).

Knowing the structure of the ERI a choice of technological subsystem variants of "assembly device" can be made. A patent search and analysis of the literature showed that when installing a seal ring is in contact with the tapered mandrel surface (basic item), this resulted in the sealing ring to be installed at a slant or in a twisted state (from arising a torque in the section). Tightening a ring in the groove increases tension on its surface, which leads to increased wear of the seal assembly and the system failure. The presence of levers and the punch in the device, gripping the sealing ring and feeding it into the hole of the basic item may cause jamming of the sealing ring between the levers (punch) and a tapered mandrel (basic item), and this will lead to cross-sectional deformation, which ultimately leads to a decrease in the quality of assembling products. Therefore, the next most suitable structural variant is as follows:

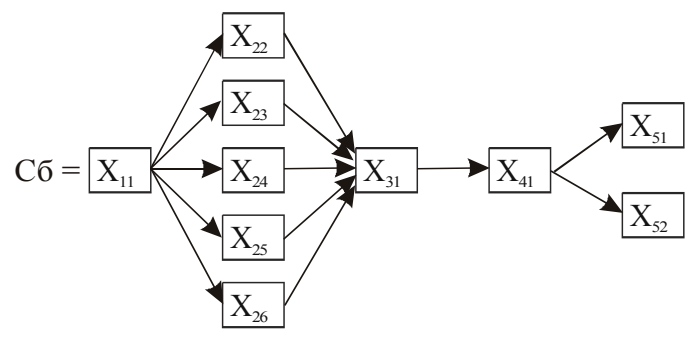

Fig. 3. The structural subsystem version "assembly mechanism"

Concerning given structure:

- the number of simultaneously mounted elements 1 ;

- type of elastic deformation of the ring item

- form deformation ( "Eight", "three-pointed star," "four-pointed star," "a multi star radiation", "projection into" "the two tabs inward");
- deforming body - levers;

- supply body - levers;

- restoration of the deformed shape of elastic ring item - either due to the elastic properties of the elastic parts, or due to the assembly of the device.

Similarly, there is variety of structural variants and other technical subsystems - assembly device for assembling of sealing compounds.

\section{Conclusion}

Thus, the morphological analysis reveals a few of the many options of rational structures. For complete information and an objective assessment of the preferred embodiment requires accounting of stochastic processes occurring in the system under real operating conditions. Conducted activities help to solve the problem at the level of synthesis of structurally combined optimization. Step parametric synthesis requires further theoretical and practical research of real structural assembly of devices that allow you to define "weak" places in the technological elements, and their improvement will lead to the eventual implementation of the most effective system.

\section{References}

1. V.M. Odrin, S.S. Kartalov, Morphological analysis systems (Kiev Naukova Dumka, 1977) (in Russian)

2. Automation of search design, Ed. by A.I. Polovinkin (M.: Radio and communication, 1981) (in Russian)

3. A.O. Kharchenko, Development of flexible production modules based on the reliability of technological systems (K.: knowledge Society, 1989) (in Russian)

4. T. Ritchey, General Morphological Analysis: A general method for non-quantified modeling. (1998)

5. T. Ritchey, "Problem Structuring using ComputerAided Morphological Analysis", Journal of the Operational Research Society (JORS), Vol. 57, No. 7, (2006)

6. T. Ritchey, Wicked Problems/Social Messes: Decision support Modelling with Morphological Analysis. Berlin: Springer, (2011)

7. F. Zwicky, \& A. Wilson (eds.), New Methods of Thought and Procedure: Contributions to the Symposium on Methodologies. (Berlin: Springer, 1967)

8. J.C. Jones, Design Methods, (Wiley, 1981)

9. Mark Sh. Levin, Modular systems design and evaluation (Springer, 2015)

10. J.C. Jones, Design Methods (Wiley, 1981).

11. Mark Sh. Levin, Composite Systems Decisions, (Springer, 2006). (in Russian)

12. Mark Sh. Levin, Combinatorial Engineering of Decomposable Systems (Kluwer, 1998). 
13. Mark Sh. Levin, Modular System Design and Evaluation (Springer, 2015). (in Russian)

14. G. S. Altshuller. To find the idea. Introduction to the theory of inventive problem solving, (Petrozavodsk: Scandinavia, 2003). (in Russian)

15. T. Baker, Attribution Standardization for Integrated Concurrent Engineering, M.S. Thesis (Brigham Young University, 2005).

16. M. Balachandran, J.S. Gero. A Knowledge-based approach to mathematical design modeling and optimization, Engineering Optimization, vol. 12, 91115 (1987).

17. J.K Novoselov, A.Yu.Tarahovsky, Justification requirements for mechanized and automated build process sealing connections with internal grooves, Scientific notes of the Crimean Engineering and
Pedagogical University, Number 27. pp. 11-16, (2011) (in Russian)

18. V.V. Burenin, V.P. Ivaniv, Estimation of durability of rubber O-rings and cuffs for rotating machines shafts mechanisms, Chem. and Naftogaz. mechanical engineering, №11, pp 32 - 33, (2003). (in Russian)

19. ISO 11346: 2004. Rubber, vulcanized or thermoplastic - Estimation of life-time and maximum temperature of use. (in Russian)

20. A.Yu. Tarahovsky, Methods deformation O-rings when installing them in the internal groove cylindrical surfaces, assembly in mechanical engineering, instrument making, Number 11. pp. 1015. (2010) (in Russian) 